\title{
LPV-based quality interpretations on modeling and control of diabetes
}

\author{
György Eigner ${ }^{1}$, József K. Tar ${ }^{2}$, Imre Rudas ${ }^{2}$, Levente Kovács ${ }^{1}$ \\ Research and Innovation Center of Óbuda University \\ ${ }^{1}$ Physiological Controls Group \\ 2 Antal Bejczy Center for Intelligent Robotics \\ Kiscelli street 82., H-1032, Budapest, Hungary \\ \{eigner.gyorgy, tar.jozsef, kovacs.levente\}@nik.uni-obuda.hu, rudas@uni-obuda.hu
}

\begin{abstract}
In this study we introduce different novel interpretations in the case of Linear Parameter Varying (LPV) methodology, which are directly usable in modeling and control design in diabetes research. These novel interpretations are based on the parameter vectors of the LPV parameter space. The theoretical solutions are demonstrated on a simple, known Type 1 Diabetes Model used in intensive care.
\end{abstract}

Keywords: Diabetes, LPV model, Affine LPV, $q L P V$

\section{Introduction}

We would like to dedicate this paper in honor of Prof. Antal Bejczy's life achievements who was a pioneer of space robotics. Prof. Bejczy provided continuous inspiration for us through his world leading work on the field of control and automation.

Modeling and control is extremely important in the research of Diabetes Mellitus (DM) [1]. In the light of the fact that no cure exists for the disease, the available treatment for those, who suffer from insulin dependent diabetes (Type 1 DM (T1DM), Double DM (DDM), etc.), the only chance in order to stay alive and/or maintain their condition, is the treatment with externally injected insulin hormone. The regular treatment is manual insulin administration by pre-calculated amounts of insulin. It depends on the assumed carbohydrate intake of the patient. More sophisticated solution is the Artificial Pancreas (AP) concept, in which the insulin injections are made by an insulin pump device with rapid acting insulin based on control algorithms and Continuous Glucose Monitoring (CGM). In recent years several advanced control solutions have appeared in literature, which may successfully deal with DM control. There were attempts with the fusion of modern and classical control theory, like switching Proportional-Integral-Derivative (PID) control [2,3], with modern control theory, for example Model Predictive Control (MPC) [4,5], 
or Soft Computing-based Control (SCC) [6,7]. Moreover, Robust Control (RC) theory also were considered by researches [8-10] in this scientific discipline. In order to test and preliminarily validate the developed algorithms in silico models can be used. The role of these mathematical models - so called patient models -, is to simulate the glucose-insulin household of a real patient as realistically as possible. Moreover, with different additional sub-models (like digestion, absorption, physical activity, sensor, noise, etc. models), highly sophisticated in silico models can be realized. Generally, one can consider large scale (macro size) models of biological processes that are relatively slowly changing [11] in time. In case of such processes Gain Scheduling Theorem (GST) can be used for modeling and control purposes. From modeling point of view, one of the most powerful tools of GST is the Linear Parameter Varying (LPV) method. LPV approaches are useful tools in control design for biological processes since their nonlinearities complicated by time delay effects. LPV techniques are frequently used in RC [12]. In order to describe a system with uncertainty, linear fractional transformation (LFT) can be used. In such cases, LPV system becomes derivable from LFT, since the nominal system depends on the uncertainties. Intuitively, this dependency can be described with function relations. Moreover, this kind of dependencies can be produced if the elements which cause the nonlinearity are getting out from the nonlinear system. In this way, a linear system is obtained which will be the function of the pulled components. Regularly, the selected elements on which the system depends are called scheduling parameters [13]. A vector can be created from them, which is called scheduling parameter vector, or shortly, parameter vector $\left(\rho \in \mathbb{R}^{k}\right)$.

In diabetes modeling uncertainty, which comes from the varying parameters, the intra- and inter-patient variability is a crucial question. Robust control allows to handle these uncertainties in a natural way. With LPV modeling linear RC methods also can be used, besides that the properties of the original nonlinear model are still valid $[8,14]$. Usually, in the physiological models the nonlinearities occur within the system model and do not affect the output matrices. This is especially true in case of diabetes modeling, since the nonlinearities are usually connected to the description of different dynamical effects, insulin kinetics, etc. That means, that if a system is described with its state-space (SS) representation the state matrix, $A(t)$ will be affected by nonlinearities. In order to hide this effect, the causing component can be selected as scheduling parameter, in this way the nonlinearities in $A(\rho(t))$ can be separated.

In this paper we are going to introduce novel interpretations and considerations about the similarity of different diabetes models. Moreover, new quality definitions will be derived based on the LPV parameter vector which can be used during modeling and controller design. 


\section{State space representations and LPV configurations}

\subsection{State space representations of a dynamic system}

A general, nonlinear time varying (NLTV) system can be represented with the following functions [15-17]:

$$
\begin{aligned}
& \dot{x}(t)=f(x(t), u(t)) \\
& y(t)=h(x(t), u(t))
\end{aligned}
$$

where $x(t) \in \mathbb{R}^{n}$ is the state vector, $f(x(t), u(t))$ is a nonlinear state function, $y(t) \in$ $\mathbb{R}^{p}$ is the output of the system and $h(x(t), u(t))$ is the nonlinear output function. With reformulation this can be described in SS form:

$$
\begin{aligned}
& \dot{x}(t)=A(t) x(t)+B(t) u(t) \\
& y(t)=C(t) x(t)+D(t) u(t)
\end{aligned}
$$

where $A(t) \in \mathbb{R}^{n \times n}$ is the state matrix, $B(t) \in \mathbb{R}^{n \times m}$ is the input matrix, $C(t) \in \mathbb{R}^{p \times n}$ is the output matrix and $D(t) \in \mathbb{R}^{p \times m}$ is the feed-forward matrix. The state matrices in (2) can be united into a single system matrix:

$$
S(t)=\left(\begin{array}{ll}
A(t) & B(t) \\
C(t) & D(t)
\end{array}\right)
$$

where $S(t) \in \mathbb{R}^{(n+p) \times(n+m)}$. Thereby (2) in simpler form via (3):

$$
\left(\begin{array}{l}
\dot{x}(t) \\
y(t)
\end{array}\right)=S(t)\left(\begin{array}{l}
x(t) \\
u(t)
\end{array}\right)
$$

If the state matrices do not depend on time, Linear Time Invariant (LTI) system occurs, described with the following SS equation:

$$
\begin{aligned}
& \dot{x}(t)=A x(t)+B u(t) \\
& y(t)=C x(t)+D u(t)
\end{aligned}
$$

and can be written in the previous compact form (4), however, here $S$ does not depend on time:

$$
\left(\begin{array}{l}
\dot{x}(t) \\
y(t)
\end{array}\right)=S\left(\begin{array}{l}
x(t) \\
u(t)
\end{array}\right)
$$

Basically, with GST an LTV system can be described with infinite number of LTI systems in continuous time domain and particular number of LTI systems in discrete time domain, if only the elements of the state matrices vary over time, but the structure of the SS representation is invariant. That means, the LTV systems run through a "trajectory" during operation over time - where the trajectory consists of infinite number of LTI systems. Fixing the elements of the SS representation of a LTV system at a given moment means that the LTV system is simplified to a LTI structure. For example, $S(t)$ exactly at $10 \mathrm{~min}$ will be equal to $S\left(t_{10}\right)=S_{10}$. 


\subsection{LPV description and configurations}

The literature distinguishes between the models according to the fact whether the selected scheduling variables are not state variables (LPV) or they are state variables also selected as scheduling parameters (quasi-LPV, qLPV). Nevertheless, there is no difference between them from notation point of view. However, the eligible interpretation of the cases is important to be noticed. In the sequel we introduce the general form of LPV systems. Assume that the parameter vector is designated with $\rho(t)$. In such a case the usual notation for an LPV model is:

$$
\begin{aligned}
& \dot{x}(t)=A(\rho(t)) x(t)+B(\rho(t)) u(t) \\
& y(t)=C(\rho(t)) x(t)+D(\rho(t)) u(t)
\end{aligned}
$$

Unification can be made similarly as in (3) and from (7):

$$
S(\rho(t))=\left(\begin{array}{ll}
A(\rho(t)) & B(\rho(t)) \\
C(\rho(t)) & D(\rho(t))
\end{array}\right) .
$$

The compact form of general LPV system from (7) becomes:

$$
\left(\begin{array}{l}
\dot{x}(t) \\
y(t)
\end{array}\right)=S(\rho(t))\left(\begin{array}{l}
x(t) \\
u(t)
\end{array}\right)
$$

The classical approaches that use LPV form in modeling apply affine and polytopic LPV system models $[13,16,17]$. However, in the recent years a soft-computing based LPV modeling issue arose the TP transformation-based LPV modeling possibility $[18,19]$. Because our quality interpretation can be used beside affine and polytopic configurations, as well, we shortly described them below.

\subsubsection{Affine LPV configuration}

In this type, the LPV systems are the affine function of the parameter vector. If the system is given with its SS representation, then it consists on two main parts: a permanent, which is independent from the parameter vector and a varying, where the dependency occurs.

$$
\begin{aligned}
& A(\rho(t))=A_{0}+\sum_{i=1}^{k} \rho_{i}(t) A_{i} \\
& B(\rho(t))=B_{0}+\sum_{i=1}^{k} \rho_{i}(t) B_{i} \\
& C(\rho(t))=C_{0}+\sum_{i=1}^{k} \rho_{i}(t) C_{i} \\
& D(\rho(t))=D_{0}+\sum_{i=1}^{k} \rho_{i}(t) D_{i}
\end{aligned}
$$

The permanent matrices are the $A_{0}, B_{0}, C_{0}, D_{0}$, which represents the independent parts from the parameter vector. The permanent and varying parts can be written in 
short form similarly as (3):

$$
S(\rho(t))=\left(\begin{array}{cc}
A_{0}+\sum_{i=1}^{k} \rho_{i}(t) \cdot A_{i} & B_{0}+\sum_{i=1}^{k} \rho_{i}(t) \cdot B_{i} \\
C_{0}+\sum_{i=1}^{k} \rho_{i}(t) \cdot C_{i} & D_{0}+\sum_{i=1}^{k} \rho_{i}(t) \cdot D_{i}
\end{array}\right)
$$

and in this way the complex system matrix can be simplified as:

$$
S(\rho(t))=S_{0}+\sum_{i=1}^{k} \rho_{i}(t) \cdot S_{i} .
$$

The affine LPV system can be written in compact form similarly as (9).

\subsubsection{Border of validity in case of affine LPV models}

The elements of the parameter vector $\rho$ forming the so-called Parameter Space (PS) which is an abstract, arbitrary mathematical space. The dimension of it is equal to the number of the selected scheduling variable.

Assume that the dimension is equal to $k$ in general case, $\rho \in \mathbb{R}^{k}$. The values of the parameter vector $\rho$ are varying over time, however, this varying is inside a particular range determined by the minimum and maximum value of the given variable. This validity range covers the meaningful parameter range based on physiological or physical processes (e.g. positive masses). This attitude determines the "Parameter Box" (PB), which is a particular region in the PS. The affine LPV models keep their validity only in this range (inside the $\mathrm{PB}$ ) during operation. This configuration is very advantageous from control engineering points of view, since

- The PB represents the workspace where the LTV system can be found during operation and each of the points also can represent an LTI system at a given moment;

- The control design tasks may be easier, because these regions are usually small;

- In case of RC, the borders of the PB can be the borders of parameter uncertainties;

- With affine LPV representation, nonlinearities can be hidden. Moreover, a given time stamp represents an LTI system, which can be selected as "operating system", if its properties are appropriate from control design point of view.

In Fig.1(a). we highlighted a $3 D$ PS, where the $\rho \in \mathbb{R}^{3}$ and the values of the scheduling parameter are varying among the range which is determined by the minimum and maximum values of the parameters. Mathematically, this can be reached, if the parameter vector is:

$$
\rho(t)=\left(\begin{array}{l}
\rho_{1}(t) \\
\rho_{2}(t) \\
\rho_{3}(t)
\end{array}\right)=\left(\begin{array}{l}
{\left[\rho_{1}^{-} . . \rho_{1}^{+}\right]} \\
{\left[\rho_{2}^{-} . . \rho_{2}^{+}\right]} \\
{\left[\rho_{3}^{-} . . \rho_{3}^{+}\right]}
\end{array}\right) .
$$




\subsubsection{Polytopic LPV configuration}

Affine LPV configuration means a natural way to describe or highlight different properties of a system, however, usually not directly used in controller design [20]. Nevertheless, the polytopic LPV configuration, which is directly derivable from affine configuration (and gives a basis for the TP-transformation based design, as well) is directly usable in such design methods. Practically, the polytopic LPV theory is based on the barycentric theorem of Möbius, describing the position of a point in a triangle with using the vertices of the triangle as reference points [21, $22]$. Further, Warren and his colleagues have proved the possibility that in case of arbitrary convex sets it is also true that with using the vertices of a convex set as reference points the position of an arbitrary internal point can be described. This is the key property which can be used in control engineering approaches [16].

As the affine LPV system is only operating inside the parameter box, the vertices of the parameter box can be used as reference points to describe each system that can occur during operation, namely, each such internal system will be the convex combination of the vertices of the polytope, if the convexity criteria is satisfied. In the case of a control system, the convexity depends on the following considerations. An internal system $S$ can be described with polytopic coordinates $\alpha_{i}$, if the system representation belonging to $\alpha_{i}$, i.e. $S_{i}$ satisfy the following restrictions:

- The polytopic coordinates should be non-negative real values, $\alpha_{i} \geq 0$;

- The sum of the polytopic coordinates should be equal to one, $\sum_{i=1}^{q} \alpha_{i}=1$;

- The internal system is the convex combination of the vertices of the polytope, $S=\sum_{i=1}^{q} \alpha_{i} S_{i}$

or shortly [15]:

$$
\left\{S=\sum_{i=1}^{q} \alpha_{i} S_{i}: \alpha_{i} \geq 0, \sum_{i=1}^{q} \alpha_{i}=1\right\} .
$$

Normally, when $q>k$ we have a redundant representation that generally allows the satisfaction of the restrictions. The barycentric coordinate function can be given in the following way [16]:

$$
" \alpha_{i}(\rho)=\frac{\Upsilon_{i}(\rho)}{\sum_{i} \Upsilon_{i}(\rho)}
$$

where $\Upsilon_{i}$ is the weight function which belongs to the ith vertex for a $\rho$ point inside the convex polytope. The weight function can be calculated as follows:

$$
\Upsilon_{i}(\rho)=\frac{\operatorname{vol}\left(\Pi_{i}\right)}{\prod_{j \in \operatorname{ind}\left(\Pi_{i}\right)}\left(n_{j} \cdot\left(\Pi_{i}-\rho\right)\right)}
$$


where $\operatorname{vol}\left(\Pi_{\mathrm{i}}\right)$ is the volume of the parallelepiped span by the normals to the facets incident on vertex $i$, i.e., $\Pi_{i},\left\{n_{j}\right\}$ is the collection of normal vectors to the facets incident on vertex $i$, and $\operatorname{ind}\left(S_{i}\right)$ denotes the set of indices $j$ such that the facet normal to $n_{j}$ contains the vertex $\Pi_{i}$. The volume of the parallelepiped can be computed as:

$$
\operatorname{vol}\left(\Pi_{i}\right)=\left|\operatorname{det}\left(n_{\text {ind }}\right)\right|
$$

where $n_{\text {ind }}$ is a matrix whose rows are the vectors $n_{j}$ where $j \in \operatorname{ind}\left(V_{i}\right)$ [16]."

Fig.1(b). shows an example whereon the aforementioned theories were taken into account in case of 3 scheduling variables. In this case, the parameter space is 3 dimensional and it is visible that the parameter box is determined by the minimum and maximum values of the parameter vector. Furthermore, the vertices of this box, $S_{i}$ serve as reference points and $\alpha_{i}$ are the convex coordinates at the same time. The actual system inside can be calculated with the barycentric calculus, namely, the actual system $S(\rho(t))$ will be the convex combination of the vertices, i.e.:

$$
S(\rho(t))=\sum_{i=1}^{8} \alpha_{i}(\rho(t)) \cdot S_{i}
$$

Obviously, if the actual system reaches a vertex that means the convex coordinate of the particular vertex will be equal to one, however, the others will be equal to zero. For example, if the system reaches the vertex $S_{1}$, the $\alpha_{1}=1$, further $\sum_{i=2}^{8} \alpha_{i}(\rho(t))$. $S_{i}=0$ and the actual system is:

$$
S(\rho(t))=\alpha_{1}(\rho(t)) \cdot S_{1}+\sum_{i=2}^{8} \alpha_{i}(\rho(t)) \cdot S_{i}=\alpha_{1}(\rho(t)) \cdot S_{1}
$$

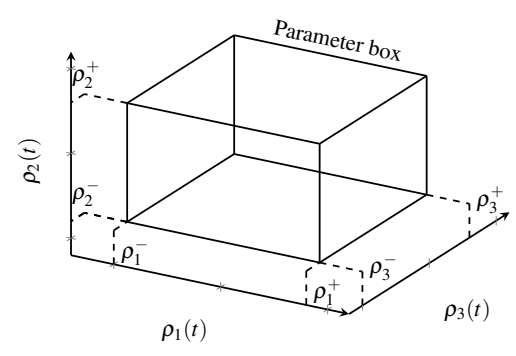

(a)

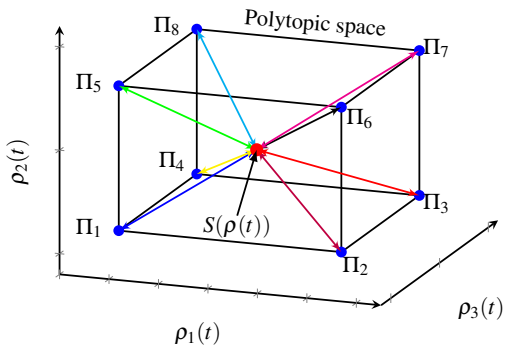

(b)

Figure 1

Affine and polytopic LPV model examples in the $3 D$ parameter space 


\subsection{Specificities of LPV models in the field of diabetes research}

According to the complex patient models the following general properties can be considered:

- Inputs are not affected by nonlinearities; they have impulse attitude; mostly consists of external insulin and glucose (or rate of appearence of glucose) intake; do not directly affect the outputs (in state space representation this means that the $D$ vector contains only zero elements and it does not depend on the parameter vector $\rho$ );

- Output(s) are connected to the blood glucose level (or the blood glucose level is the output itself); not affected by nonlinearities;

- Since the nonlinearities do not affect the inputs and the outputs, it is not necessary to select their elements as scheduling parameters, which means that $B$ and $C$ are independent from the parameter vector $\rho$; moreover, these usually do not depend on time;

- The nonlinearities occur in the state matrix $(A)$ regarding to glucose-insulin dynamics, glucose and/or insulin absorption, effect and dynamics of insulin; the intra- and inter-patient variabilities are represented in the elements of $A$ and usually these are time dependent; scheduling variables should be selected from the elements of $A$ in order to hide the nonlinearities and make the handling of $A$ convenient from control point of view.

From the aforementioned consideration it can be derived that the LPV-type diabetes models have the following form which can be observed in several studies $[8,9,14]$ :

$$
\begin{aligned}
& \dot{x}(t)=A(\rho(t)) x(t)+B u(t) \\
& y(t)=C x(t)+D u(t)
\end{aligned}
$$

where the system matrix $S$ is the following:

$$
S(\rho(t))=\left(\begin{array}{cc}
A(\rho(t)) & B \\
C & D
\end{array}\right)
$$

and the state space representation in compact form is:

$$
\left(\begin{array}{l}
\dot{x}(t) \\
y(t)
\end{array}\right)=S(\rho(t))\left(\begin{array}{l}
x(t) \\
u(t)
\end{array}\right)
$$

Equation (21) shows that in this form the LPV-type diabetes models only contain dependency from the parameter vector $\rho$ in the state matrix $A$ and all time dependent components are selected as scheduling variable. 


\section{Different interpretations of quality based on LPV con- figurations}

\subsection{Norm-based "difference" definition in the Parameter Space}

Each dimensions of the PS correspond to an element of the parameter vector ( $\rho \in$ $\mathbb{R}^{k}$ ). Inside this abstract space each point can be determined by the corresponding parameter vector. Furthermore, this abstract PS is an Euclidean vector space and vector $L_{p}$ norms can be interpreted. Assume two vectors $\rho_{a}, \rho_{b} \in \mathbb{R}^{k}$ in the PS. The $L_{2}$ norm based distance between the vectors can be described as follows:

$$
\left\|\rho_{a}-\rho_{b}\right\|_{2}=d_{2} \text {. }
$$

The defined $d_{2}$ can be used in various way depending on the interpretation of the PS which are presented in the next section.

\subsection{Possible interpretations of the defined norm-based differ- ence in the Parameter Space}

The points of the PS which are determined by the parameter vector can be interpreted on their own as vectors, which elements consist of the parts of the system. However, the parameter vectors can unequivocally determine an underlying LTI system in the affine LPV case and a well-characterized LTI system in the polytopic case. The following statements are general LPV model properties regardless it is the affine or polytopic type LPV model.

When the goal is to emphasize particular properties of a system, each of the parts representing these properties have to be selected as scheduling variables. For example, if we investigate only the insulinemia (I) and blood glucose (G) from (A-1), these have to be selected as scheduling variables and the PS will be 2 dimensional. In this case, the introduced $d_{2}$ is appropriate to define a "difference" between the $I$ and $G$ which belong to different points in the PS. Namely, a permanent reference parameter vector $\rho_{\text {ref }}$ can be defined with given constant $I$ and $G$. The actual parameter vector $\rho_{\text {actual }}$ varies over time. In this case $d_{2}=\left\|\rho_{\text {ref }}-\rho_{\text {actual }}\right\|_{2}$ determines the 2-norm based difference of them and this can be interpreted as an "error" or "quality" signal, if $\rho_{\text {ref }}$ and $\rho_{\text {actual }}$ are different during operation. Generally, this interpretation can be extended for any $k$ dimensional parameter vector.

If the question is to design a controller the key point is what the selected scheduling variables are. At this point several approaches and interpretations can be distinguished. The main ones are the following:

1. The selected scheduling variables are those properties which have to be monitored during the operation. In this case, the 2-norm based difference can be used as "quality" signal and the performance of different controllers can be compared with this quality signal in the PS.

2. The selected scheduling variable are those properties which have to be controlled and monitored during operation. In this case, we can interpret the 
2-norm based signal as "error" signal. This type of error can be used during the controller design.

3. The selected scheduling variables are those components which are time dependent in LTV case. The parameter vector unequivocally determines the underlying LTI systems which can occur from the general LTV system during operation at given time moments. In this case, the defined 2-norm difference can be used as "metric" in order to compare LTI systems in the PS.

4. The selected scheduling variables are those components which are causing nonlinearities in NLTV case. The general purpose of this approach is that the linear controller design techniques become usable beside polytopic LPV systems. If each nonlinearity causing and time dependent parameters are selected as scheduling variables, the parameter vector unequivocally (affine LPV case) or satisfactorily (polytopic LPV case) determines an LTI system during operation at given time moments. In this case the defined 2-norm difference can be used as metric in order to compare LTI systems in the PS.

In the following sections we detail the key aspects from the above mentioned points.

\subsubsection{The 2-norm based difference as quality and error signal}

The most important issue in these cases is the way how the parameters are selected from the original model and the interpretation of them.

If the scheduling parameters are selected each-by-each and not grouped, then each dimensions of the PS will be an individual variable with physiological or physical meaning (e.g. the $I$ and $G$ parameter from (A-1)).

However, the scheduling variables can be grouped (e.g.,(25)). In this case the scheduling variables can loose their original meaning and cannot be interpreted individually.

Nevertheless, the grouped scheduling parameters allow to interpret the 2-norm based difference sophisticatedly. If the goal is to monitor how the specific properties of the system vary over time and compare this vary with predefined requirements, the 2-norm based difference can be interpreted as quality signal. This approach can be important in such application, where the different parts of the system cannot change to drastically relative to each other. Naturally, in this case, these specific parts have to be selected as scheduling variables.

It has to be noted that the observability should be considered in this case, since, the selected parts have to be observable or estimable. Fig. 2(a). shows a 2 dimensional parameter space. For example, a possible goal (beside other goals) of the applied controller can be to hold permanently two system properties during operation. If these properties (which are represented with different parts of the system) are selected as scheduling variables, the performance of the controller can be assessed based on the $d_{2}(t)$ signal.

During controller design the 2-norm based difference can be used as an "error" signal in the classical meaning. The appropriate selection and interpretation of the scheduling variables are necessary. The observability and controllability of the scheduling variables are important issues, as well. 
The first step is the selection of parts of the models as scheduling variables which have to be controlled. However, in case of grouped multiply out (e.g. $S_{I} Q(t) /(1+$ $\left.\alpha_{G} Q(t)\right)$ of (25) ) should be reasonable. The error signal ought to be known at every time moment as the basis of control.

If the elements of the parameter vector are not observable, they have to be estimated or approximated. The control signal affects the scheduling variables directly or through coupling. Without connection, the scheduling variables cannot be influenced by the control signal.

Beside these constraints, controller design is possible. For example, the recently developed Robust Fixed Point Transformation (RFPT)-based controller design methodology can be used [23]. The first stage is the investigation of the effect chain of the control action, namely, how the control signal affects the controlled variables which are here the scheduling variables $\rho(t)=f\left(\rho(t)^{-}, u(t)\right)$, where $\rho(t)^{-}$is the a-priori knowledge about the $\rho(t)$ and $u(t)$ is the control signal. With approximate inverse kinematic description $\left(\tilde{u}(t)=f\left(\rho(t), \rho(t)^{-}\right)\right)$and appropriate control laws a RFPTbased controller can be designed. In this case the error signal can be the developed by 2-norm based difference $\left(d_{2}\right)$, which arises when the nominal prescriptions of the controlled variables (the scheduling variables) are not equal with the actual values of them. Geometrically, the nominal prescriptions of the controlled variables can be a permanent point of the PS $\left(\rho_{\text {ref }}\right)$ and the actual values $\rho_{a c t}(t)$ are varying in time during operation. Based on the arised error signal $d_{2}(t)$ a RFPT-based controller can be designed [23].

In Fig.2(a). a $2 D$ example can be seen, where $d_{2}(t)$ can be interpreted as the mentioned error signal. The comparability of the order of magnitudes of the scheduling variables represent a significant point. The nature of the Euclidean norms determine the particular difference signals affect mostly on the 2-norm based difference which has the highest magnitudes (e.g. the $\rho_{\text {reference }, 1}-\rho_{\text {actual }, 1} \gg \rho_{\text {reference }, k}-\rho_{\text {actual }, k}$, $\rho \in \mathbb{R}^{k}, k \neq 1$ determines that the 2-norm based difference will have strong connection with the variation of $\left.\rho_{\text {reference }, 1}-\rho_{\text {actual }, 1}\right)$. If the scheduling variables have to be considered with the same "weight" (they have the same importance), different normalization and weighting techniques can be used [20].

\subsubsection{The 2-norm based difference as comparison of systems}

Generally, LPV techniques are used in order to embed the uncertainties into a system model or hide the system model nonlinearities by making the application of linear controller design techniques possible. LPV models can be used in the classical control design solutions, however, the use of such models according to Linear Matrix Inequality (LMI) based controller design methodologies are possible, as well. LMIs are powerful mathematical opportunities of controller design techniques.

Almost each control design method can be formulated as a LMI problem and can be solved via iterative numerical processes [17]. In recent years parallel with numerical computational evolution a wide range of LMI applications were discovered and used in control engineering [16,24].

However, the basic concept behind the LPV-LMI based modeling and control approaches consist in guaranteeing and exploiting the convexity properties. Basically, 
this means that it is enough to design such sub-controllers which can deal with the LTI systems in the vertices of the convex polytope, and the convex combination of such controllers can handle each occurring LTI system during operation, if the basic LPV model was appropriate.

In order to use the developed 2-norm based difference as a "metric" on the underlying systems which are determined by parameter vectors inside the PS, several control and mathematical constraints have to be considered. Particular parameter vectors belong to each of the points inside the PS. Since, the parameter vector consist of elements which were multiplied out from the SS model, a parameter vector can determines an underlying system. The key questions are the type of the underlying systems regarding to the parameter vector and how can the parameter vector be used to describe differences among the underlying systems. A few scenarios can be considered dependent on the type of the original and the describing LPV system. The reasonable original system can be NLTV, LTV and LTI beside the describing LPV system (affine or polytopic).

- In LTI-LPV case, each of the points inside the PS is an LTI system and fully determined by the parameter vector.

- In LTV case, a parameter vector determines the underlying system only, if each time-dependent element is selected as scheduling variable.

- In NLTV case, if each time-dependent and nonlinearity causing element is selected as scheduling variable, the parameter vector determines the underlying system.

In all three cases, the parameter vectors determine an underlying LTI system. In NLTV-LTV-LPV case, the original models become simpler. Furthermore, during operation these get around a path inside the PS. The most typical application is when the nonlinearity causing elements are selected as scheduling variables from the NLTV system and the obtained LPV model is used in a LPV-LMI control application. However, in this case, a parameter vector does not determine equivocally the underlying system, since, the time-dependent components can cause hidden differencies, which cannot be seen through the parameter vector.

Assume that the selection of the scheduling variables was appropriate and each parameter vector determines an underlying LTI system. That means the parameter vector-based differences can be interpreted as a "metric" on the occurring LTI systems for which these vectors belong. Namely, instead of the Frobenius-norm based difference in the systems' space the Euclidean-norm based difference in the PS can be used to determine the "difference" between the occurring LTI systems.

$$
\left\|S\left(\rho_{a}\right)-S\left(\rho_{b}\right)\right\|_{F} \rightarrow\left\|\rho_{a}-\rho_{b}\right\|_{2}=d_{2} .
$$

In the convex polytope, every LTI systems are uniquely specified with their parameter vectors as a consequence of the aforementioned statements. However, the LTI systems in the convex polytope can be calculated as the convex combination of the vertices of the convex polytope. The key question then is the determination of barycentric coordinates $\left(\alpha_{i} \in \mathbb{R}^{q}\right)$ via the uniqueness of the vertices of the given polytope. 
Inside the convex polytope each occurring LTI system is over defined, since in a $k$ dimensional parameter space a $q$ pieces coordinate is used to define them, where $q>k$. That means, if the barycentric coordinates are arbitrarily defined, the occurring LTI system description will not be unequivocal, since with the same set of coordinates describes more then one system. In other words, because of the null space problem (differences could occur in the null spaces of the given LTI system) the parameter vector based metric cannot be used as a classic "metric" and cannot be unequivocally interpreted on the LTI systems behind.

Nevertheless, the calculation of the barycentric coordinates $\left(S=\sum_{i=1}^{q} \alpha_{i} S_{i}\right)$ are connected to the selected vertices of the convex polytope and equivocally defined by 15-17. With this condition, the defined metrics can be valid on the occurring LTI systems in case of polytopic LPV systems, as well.

The developed parameter vector based metric can be used in modeling and control as a "quality marker". For example, if a given LPV model is used during identification, the identified model $S\left(\rho_{\text {ident }}\right)$ can be compared to a reference model $S\left(\rho_{\text {ref }}\right)$ in order to estimate the efficiency of the identification procedure. Furthermore, this can be an on-line estimation as well, when the system under identification is described with $S\left(\rho_{\text {actual }}\left(t_{p}\right)\right)$. This procedure can be characterized by the developed $\left\|d_{2}\right\|_{2}(t)$ instead of the Frobenius-norm based difference. If the goal is to monitor the variation of the system during operation compared to a reference system, the previous solution can be used here as well. Further usabilities and interpretations will be developed in our future work.

Fig.2(b). shows a $2 D$ example about the aforementioned interpretations. The PB is the rectangle which is determined by the $\rho_{\min , 1,2}$ and $\rho_{\max , 1,2}$ in the PS. Further, this is the validity border of the affine LPV model. At the same time, the rectangle forms a convex polytope. Inside the polytope each occurring LTI system can be calculated as the convex combination of the vertices of the convex polytope. Each aforementioned interpretations and issues are demonstrated on a biomedical engineering example regarding diabetes.

\section{Example of the developed approach: Modeling of di- abetes}

\subsection{Selected diabetes model and an LPV form of it}

We have selected a simple diabetes model developed for the Intensive Care Unit (ICU) treatment by Wong et al. in [25,26]. The model is a 3rd order one described by (A-1). The model equations can be handled as in (20-22), which means only the state matrix depends on the parameter vector $(A(\rho(t)))$. The main aspect of the model is to describe the glucose-insulin dynamics of an inpatient who suffers from T1DM and is nurtured on the Intensive Care Unit (ICU) [25,26]. It is expected that this simple model -after preliminary identification-, can provide the current and the future Blood Glucose (BG) level of the patient with a precision that is good enough for the realization of the tight glycemic control (TGC). Detailed description of the 


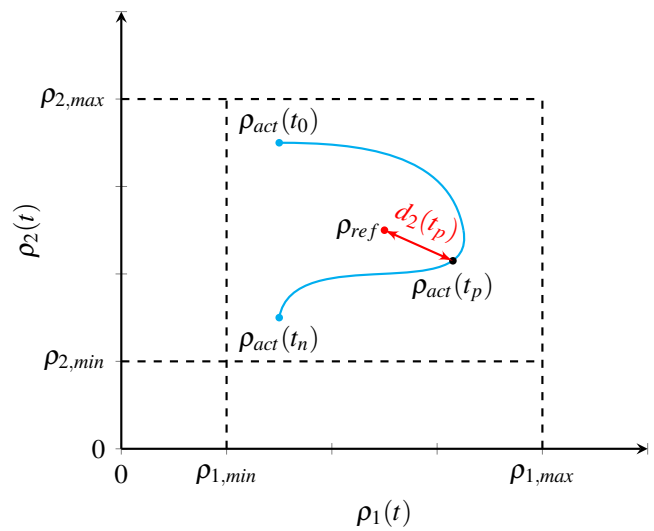

(a)

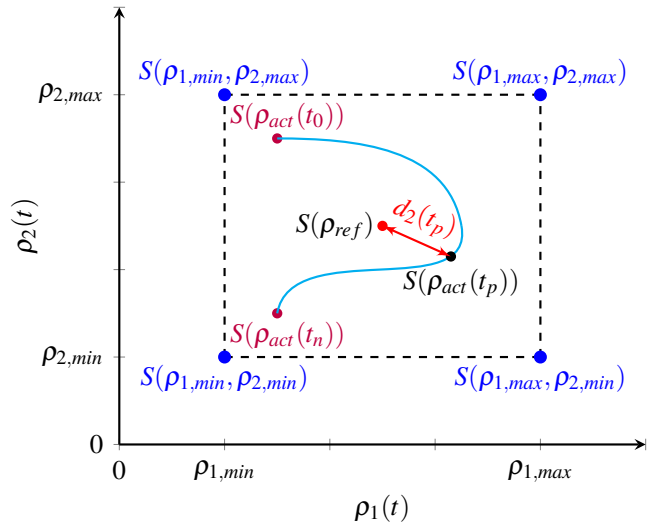

(b)

Figure 2

Examples of the possible interpretation of the 2-norm based difference

model can be found in the Appendix of the current paper.

\subsection{1 qLPV model of the original Wong model}

We selected the following scheduling parameters as the elements of the parameter vector based on our previous work [27]:

$$
\rho(t)=\left[\begin{array}{l}
\rho_{1}(t) \\
\rho_{2}(t) \\
\rho_{3}(t)
\end{array}\right]=\left[\begin{array}{c}
\frac{S_{I} Q(t)}{1+\alpha_{G} Q(t)} \\
\frac{S_{I} G_{E}}{1+\alpha_{G} Q(t)} \\
\frac{1}{1+\alpha_{I} I(t)}
\end{array}\right] .
$$


It can be seen, that the $\rho(t)$ contains grouped variables. The goal here was to use this LPV model in classical LPV-LMI controller design. Each nonlinearity causing element was selected as scheduling variable. Based on (A-1 and 25), the qLPV model of the original Wong model can be described as follows:

$$
\begin{aligned}
& A(\rho(t))=A_{0}+A_{1} \rho_{1}(t)+A_{2} \rho_{2}(t)+A_{3} \rho_{3}(t)= \\
& {\left[\begin{array}{ccc}
-p_{G} & 0 & 0 \\
0 & -k & k \\
0 & 0 & 0
\end{array}\right]+\left[\begin{array}{ccc}
0 & -1 & 0 \\
0 & 0 & 0 \\
0 & 0 & 0
\end{array}\right] \rho_{1}(t)} \\
& +\left[\begin{array}{ccc}
0 & -1 & 0 \\
0 & 0 & 0 \\
0 & 0 & 0
\end{array}\right] \rho_{2}(t)+\left[\begin{array}{ccc}
0 & 0 & 0 \\
0 & 0 & 0 \\
0 & 0 & -n
\end{array}\right] \rho_{3}(t) \\
& B(\rho(t))=\left[\begin{array}{lll}
1 & 0 & 0 \\
0 & 0 & 0 \\
0 & \frac{1}{V_{L}} & -p_{4} I_{b}
\end{array}\right] \\
& C(\rho(t))=\left[\begin{array}{lll}
1 & 0 & 0
\end{array}\right]
\end{aligned}
$$

After defining the border of the parameter box, namely the convex polytopic space, the polytopic model form of the qLPV model can be easily obtained based on the affine qLPV form of (26). We have selected tight ranges in every dimension in order to catch the dynamics as precise as possible:

$$
\rho(t)=\left(\begin{array}{l}
\rho_{1}(t) \\
\rho_{2}(t) \\
\rho_{3}(t)
\end{array}\right)=\left(\begin{array}{l}
{\left[\rho_{1}^{-} . . \rho_{1}^{+}\right]} \\
{\left[\rho_{2}^{-} . . \rho_{2}^{+}\right]} \\
{\left[\rho_{3}^{-} . . \rho_{3}^{+}\right]}
\end{array}\right)=\left(\begin{array}{l}
{[0 . .5]} \\
{[0 . .5]} \\
{[0 . .5]}
\end{array}\right) .
$$

\subsection{Presentation of the results}

The main goal was to test the usability of the developed "metric" without physiological constraints. In this demonstration we used the consideration of Sec. 3.2.2.

Fig.3. shows the changing of the elements of the parameter vector $\rho(t)$ and the developed 2-norm based difference. On every diagram, the dashed line represents the fixed value, which belongs to the $\rho_{\text {ref }}=\left[\begin{array}{lll}0.2, & 0.01025, & 0.98\end{array}\right]^{T}$. It can be seen regarding to the input selected as a symmetrical repeating impulse $(P(t)=100$ at every $140 \mathrm{~min}$ for $7 \mathrm{~min}$ long and $u_{e x}(t)=100$ at every $130 \mathrm{~min}$ for $6.5 \mathrm{~min}$ long) that after the first period's decay, the parameter vector have taken the same values in each cycle, which means the same LTI systems occur over time in each cycle.

Fig.4. shows the same signals as Fig.3. on one diagram in order to compare the orders of magnitudes. It can be seen that based on Sec. 3.2.1 those signals that reflect mostly in the $\left\|d_{2}\right\|_{2}$ have the highest amplitude. Since, in this case the $\rho_{1}(t)$ has the highest amplitudes, the $\left\|d_{2}\right\|_{2}$ correlated mostly with $\rho_{1}(t)$. If each scheduling variables have to be considered with the same weight, normalization procedures 
can be done [20]. However, we did not apply such methods as the goal was only demonstration.

If the parameter vectors fully determine the underlying LTI systems during operation, the parameter vector based metric can be used to compare the "difference" between these systems. Fig.5. shows this issue in case of the selected model and parameter vector, namely, instead of the Frobenius-norm based "difference" the developed metric can approximately provides similar results. Naturally, the signals are not the same, since the numerical computations are different. However, the maximum root mean square error (RMSE) was $5.4 e^{-5}$.
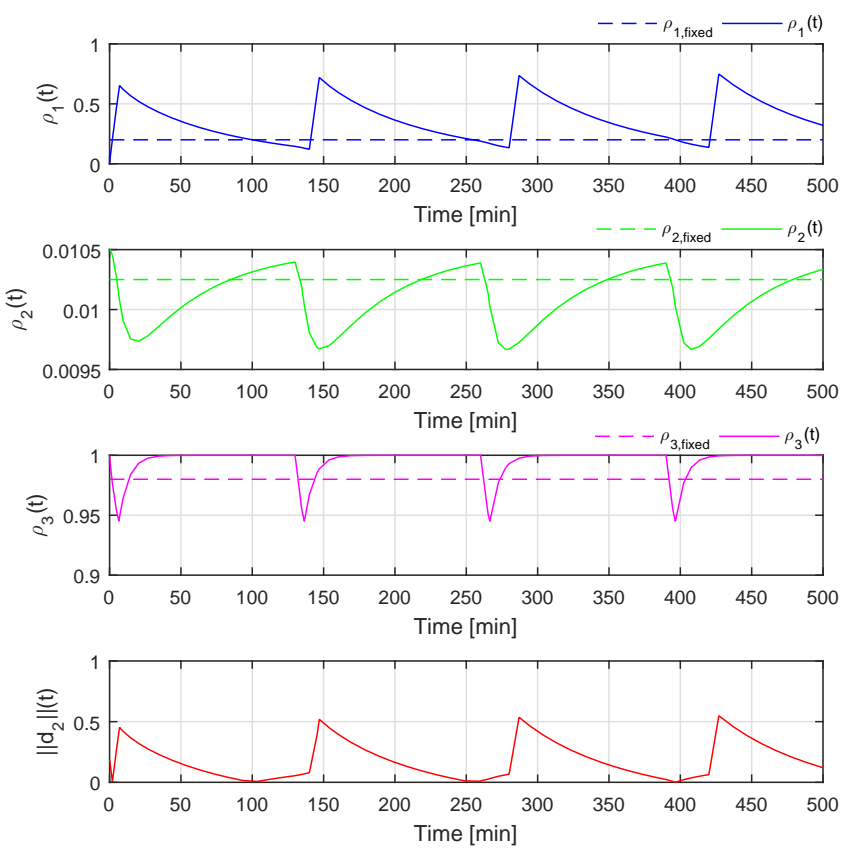

Figure 3

Varying of the scheduling variables and the norm-based error signal

\section{Conclusions}

In this study we introduced norm based "difference" interpretation regarding to LPV systems, based on the properties of the LPV parameter space. We have defined how to use these interpretations as error and quality criteria during modeling and control and demonstrated our theoretical findings by a concrete example on diabetes modeling and control of ICU patients. Our future work will focus on the investigation of how this approach can be implemented to the actual LMI based control design methods in order to realize more precise controller for the practice. 


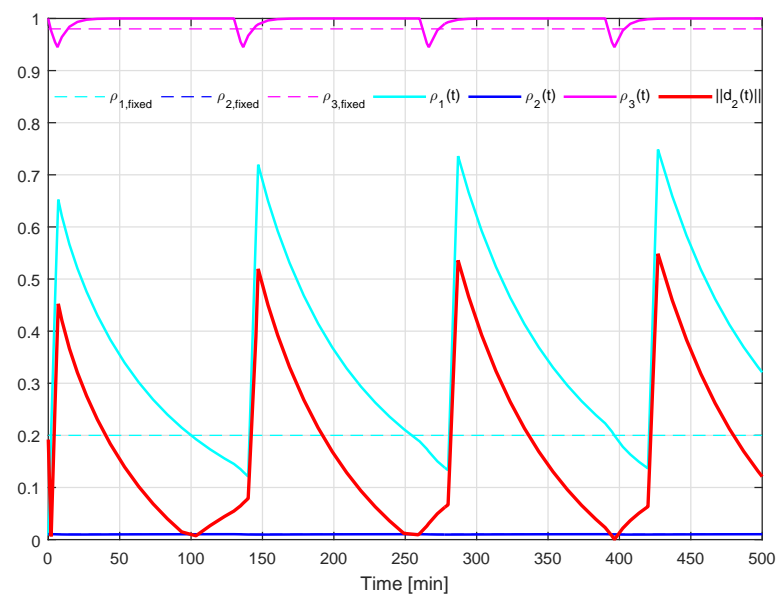

Figure 4

Comparison of the magnitudes of the scheduling variables and the norm-based error signal

\section{Acknowledgement}

Gy. Eigner thankfully acknowledge the support of the Robotics Special College of Obuda University. L. Kovács is also supported by the János Bolyai Research Scholarship of the Hungarian Academy of Sciences. The research was also supported by the Research and Innovation Center of Obuda University.

\section{References}

[1] V.N. Shah, A. Shoskes, B. Tawfik, and S.K. Garg. Closed-loop system in the management of diabetes: Past, present, and future. Diabetes Technol The, 16(8):477 - 490, 2014.

[2] S. Kamath. Model based simulation for type 1 diabetes patients. Asian Am J Chem, 1(1):11 - 19, 2013.

[3] G. Marchetti, M. Barolo, L. Jovanovic, H. Zisser, and D.E. Seborg. An improved PID switching control strategy for type 1 diabetes. IEEE T Bio-Mde Eng, 55:857 - 865, 2004.

[4] G. Schlotthauer, L.G. Gamero, M.E. Torres, and G.A. Nicolini. Modeling, identification and nonlinear model predictive control of type i diabetic patient. Med Eng Phys, 28:240 - 250, 2006.

[5] P. Maxime, H. Gueguen, and A. Belmiloudi. A robust receding horizon control approach to artificial glucose control for type 1 diabetes. Nonlin Contr Sys, 9(1):833 - 838, 2013. 


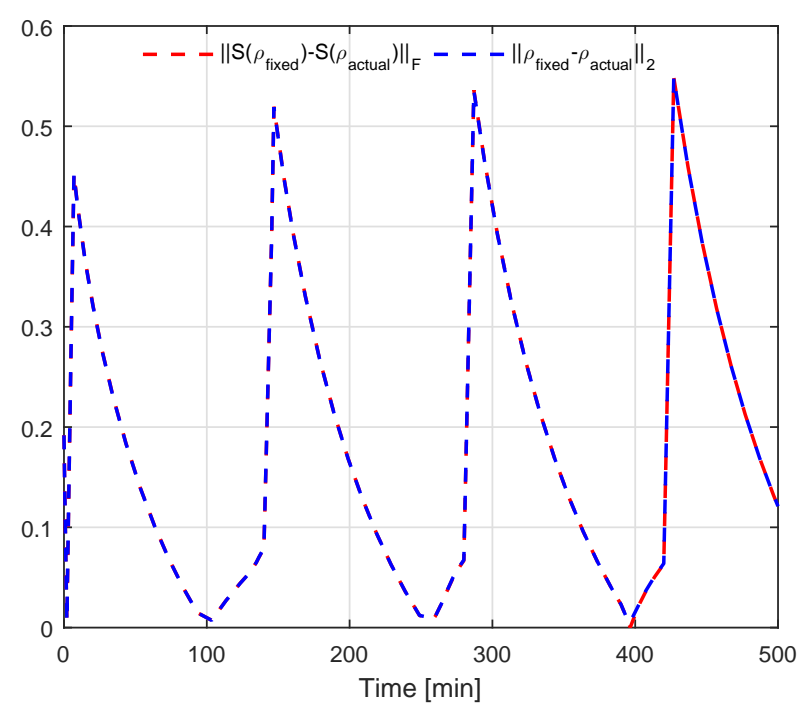

Figure 5

Different norm-based differences

[6] P. Herrero, P. Georgiou, N. Oliver, D.G. Johnston, and C. Toumazou. A bioinspired glucose controller based on pancreatic $\beta$-cell physiology. J Diabetes Scien Technol, 6(3):606-616, 2012.

[7] E. Atlas, R. Nimri, S. Miller, E.A. Grunberg, and M. Phillip. Md-logic anrtificial pancreas system. a pilot study in adults with type 1 diabetes. Diab Care, 33:1072-1076, 2010.

[8] P. Szalay, Gy. Eigner, and L. Kovács. Linear matrix inequality-based robust controller design for type-1 diabetes model. In IFAC 2014 - 19th World Congress of The International Federation of Automatic Control, pages 9247 - 9252. IFAC, 2014.

[9] L. Kovács, P. Szalay, Zs. Almássy, and L. Barkai. Applicability results of a nonlinear model-based robust blood glucose control algorithm. J Diabetes Scien Technol, 7(3):708 - 716, 2013.

[10] P. Colmegna and S. Peña. Analysis of three T1DM simulation models for evaluating robust closed-loop controllers. Comput Meth Prog Bio, 113(1):371 $-382,2014$.

[11] S.S. Hacisalihzade. Biomedical Applications of Control Engineering. Springer-Verlag, Berlin, 1st edition, 2013.

[12] L. Kovács, B. Benyó, J. Bokor, and Z. Benyó. Induced $l_{2}$-norm minimization of glucose-insulin system for type I diabetic patients. Comput Meth Prog Bio, 102(2):105 - 118, 2011. 
[13] C. Scherer and S. Weiland. Lecture Notes DISC Course on Linear Matrix Inequalities in Control. Delft University, 1999.

[14] P. Szalay, Gy. Eigner, M. Kozlovszky, I. Rudas, and L. Kovács. The significance of LPV modeling of a widely used T1DM model. In EMBC 2013 35th Annual International Conference of the IEEE Engineering in Medicine and Biology Society, pages 3531 - 3534. IEEE EMBS, 2013.

[15] D.W. Gu, P.H. Petkov, and M.M. Konstantinov. Robust Control Design with Matlab. Springer, London, 2nd edition, 2013.

[16] A.P. White, G. Zhu, and J. Choi. Linear Parameter Varying Control for Engineering Applicaitons. Springer, London, 1st edition, 2013.

[17] O. Sename, P. Gáspár, and J. Bokor. Robust control and linear parameter varying approaches, application to vehicle dynamics. volume 437 of Lecture Notes in Control and Information Sciences. Springer-Verlag, Berlin, 2013.

[18] B. Takarics and P. Baranyi. TP model-based robust stabilization of the 3 degrees-of-freedom aeroelastic wing section. ACTA Polytech Hung, 12(1):209 $-228,2015$.

[19] B. Takarics and P. Baranyi. Friction compensation in TP model form - aeroelastic wing as an example system. ACTA Polytech Hung, 12(4):127 - 145, 2015 .

[20] W.S. Levine. The Control Engineering Handbook. CRC Press, Taylor and Francis Group, Boca Raton, 2nd edition, 2011.

[21] F.A. Möbius. The Barycentric Calculus (in German). Verlag von Johann Ambrosius Barth, Leipzig, 1st edition, 1827.

[22] J. Fauvel, F. Raymond, and R. Wilson, editors. Möbius and his Band. Oxford University Press, Oxford, 1st edition, 1993.

[23] J.K Tar, J.F Bitó, L. Nádai, and J.A.T Machado. Robust Fixed Point Transformations in Adaptive Control Using Local Basin of Attraction. ACTA Pol Hung, 6(1):21-37, 2009.

[24] S. Boyd, L. El Ghaoui, E. Feron, and V. Balakrishnan. Linear Matrix Inequalities in System and Control Theory. SIAM Studies in Applied Mathematics. SIAM, Philadelphia, 1st edition, 1994.

[25] X.W. Wong, J.G. Chase, G.M. Shaw, C.E. Hanna, T. Lotz, J. Lin, I. SinghLevett, L.J. Hollingsworth, and O.S.W. Wong. Model predictive glycaemic regulation in critical illness using insulin and nutrition input: A pilot study. Med Eng Phys, 28:665 - 681, 2006.

[26] X.W. Wong, J.G. Chase, G.M. Shaw, C.E. Hann, J. Lin, and T. Lotz. Comparison of adaptive and sliding-scale glycaemic control in critical care and the impact of nutritional inputs. In 12th International Conference On Biomedical Engineering, pages 1-4, 2005. 
[27] L. Kovács, A. György, B. Kulcsar, P. Szalay, B. Benyo, and Z. Benyo. Robust control of type 1 diabetes using $\mu$-synthesis. In UKACC International Conference on Control 2010, pages 1 - 6, 2010.

\section{Appendix}

The Wong-model consist of the following equations $[25,26]$ :

$$
\begin{aligned}
& \dot{G}(t)=-p_{G} G(t)-S_{I}\left(G(t)+G_{E}\right) \frac{Q(t)}{1+\alpha_{G} Q(t)}+P(t) \\
& \dot{X}(t)=-k I(t)-k Q(t) \\
& \dot{I}(t)=-\frac{n I(t)}{1+\alpha_{I} I(t)}+\frac{u_{e x}(t)}{V}
\end{aligned}
$$

The following table contains the parameters, their descriptions and their values which were used in this study regarding to the Wong-model $[25,26]$.

Table A-1

Detailed desriptions and values of the parameters of the Wong-model

\begin{tabular}{|c|c|l|c|}
\hline Notation & Unit & Description & Value \\
\hline$G$ & $m m o l / L$ & $\begin{array}{l}\text { Plasma glucose above equilibrium } \\
\text { level }\end{array}$ & - \\
\hline$Q$ & $m U / L$ & $\begin{array}{l}\text { Concentration of insulin bounded to } \\
\text { interstitial sites }\end{array}$ & - \\
\hline$I$ & $m U / L$ & $\begin{array}{l}\text { Plasma insulin resulting from exter- } \\
\text { nal input }\end{array}$ & - \\
\hline$P$ & $m m o l / L / m i n$ & Total plasma glucose input & - \\
\hline$u_{e x}$ & $m U / m i n$ & External insulin input & - \\
\hline$G_{E}$ & $m m o l / L$ & Plasma equilibrium level & 10.5 \\
\hline$p_{G}$ & $1 / m i n$ & Endogenous glucose clearance & 0.01 \\
\hline$S_{I}$ & $L / m U / m i n$ & Insulin sensitivity & 0.001 \\
\hline$V$ & $L$ & Insulin distribution volume & 12 \\
\hline$k$ & $1 / m i n$ & $\begin{array}{l}\text { Effective life of insulin in the com- } \\
\text { partment }\end{array}$ & 0.0198 \\
\hline$n$ & $1 / m i n$ & First order decay rate from plasma & 0.16 \\
\hline$\alpha_{I}$ & $L / m U$ & Plasma insulin disappearance & 0.0017 \\
\hline$\alpha_{G}$ & $L / m U$ & Insulin effect & 0.0154 \\
\hline
\end{tabular}

